\title{
Child Torture as a Form of Child Abuse
}

\author{
Barbara Lou Knox ${ }^{1}$ - Suzanne P. Starling ${ }^{2}$ - Kenneth W. Feldman ${ }^{3}$ - Nancy Kellogg ${ }^{4}$. \\ Lori Frasier $^{5} \cdot$ Suzanna Tiapula ${ }^{6}$
}

Published online: 13 July 2016

(C) Springer International Publishing 2016

To the Editor:

The authors thank Doctors Alexander and Pena for their comments regarding our manuscript "Child Torture as a Form of Child Abuse". When we wrote about Torture as Form of Child Abuse, we attempted to develop a coherent medical definition for this under-appreciated form of abuse. Fundamentally, we observed that the torture of the children in our case series was a means of systematically demeaning, subjugating and controlling the victims. Various forms of poly-victimization were used by perpetrators of all of our cases including both physical and psychological abuse. Our definition reflects the predominant forms of poly-victimization documented in this case series.

As noted by Alexander and Pena, we reported that sexual abuse was also used as a form of poly-victimization in the abusive torture of some of the children in this case series.

As authors, we discussed the complexities of the sexual torture of children (both from a power/control and ritualistic

Barbara Lou Knox

blknox@pediatrics.wisc.edu

1 University of Wisconsin School of Medicine and Public Health, American Family Children's Hospital, Madison, WI, USA

2 Eastern Virginia Medical School, Children's Hospital of The King's Daughters, Norfolk, VA, USA

3 University of Washington, and Seattle Children's Hospital, Seattle, WA, USA

4 University of Texas Health Science Center at San Antonio, San Antonio, TX, USA

5 Penn State Hershey Medical Center, Penn State College of Medicine, Hershey, PA, USA

6 Institute on Violence, Abuse \& Trauma, San Diego, CA, USA aspect). We chose to exclude cases in which the primary motivation seemed to be sexual rather than control and subjugation. The authors noted that the sexual torture of children had this fundamentally different motivation than what we were addressing in our case series. Although isolation of the victim is common both in the torture we reported and sexual torture, in the latter case it is primarily used to avoid detection and to maintain sexual access. As Alexander and Pena noted, the literature has never adequately addressed this issue; some of the current authors plan to separately analyze and define this type of primary abuse. Internet and communication technology facilitated crime components are becoming a frequent tool for this type of torture maltreatment and will be further analyzed. Several of our article's authors have been involved in the medical treatment of victims of sexual torture in childhood. We do note, however, that often physical, psychological and sexual tortures co-occur in these cases.

As authors, we analyzed the issue of whether a torture definition in child abuse required repeated events over a prolonged period or if an extended act would constituted the torture of a child. The authors clearly observed cases where the same issues arose in a single, somewhat extended period of severe abuse. In response to Alexander and Pena's second comment, the wording of "permanent bodily dysfunction" was made because most of the children we studied had permanently disfiguring or impairing injuries. Having frequently worked with the court system, we realize that "prolonged" would also be open to wildly different legal interpretations. We proposed our definition as a starting point, hoping it would stimulate discussion, and possibly modification, to better deal with this problem. Should language which would be both legally explicit, but still flexible enough to encompass the range of these cases be proposed, we would consider modifying the definition. 